\title{
RESEARCH PAPER \\ ASSESSMENT OF FRICTION BETWEEN A ROLLING CYLINDRICAL ELEMENT AND A DEFORMABLE FLAT SURFACE
}

\author{
F. Davis ${ }^{1}$, P. Y. Andoh ${ }^{1}$, M. N. Sackey ${ }^{1}$ and S. P. Owusu-Ofori ${ }^{2}$ \\ ${ }^{I}$ Department of Mechanical Engineering, KNUST, Kumasi, Ghana. \\ ${ }^{2}$ Department of Mechanical Engineering, North Carolina A\&T State University, Greensboro, \\ North Carolina, USA.
}

\begin{abstract}
A cost-effective friction coefficient measuring technique was developed and tested. The technique involved the mounting of two sensing elements on the surface of rolls, in a manner that sought to measure simultaneously the normal and the tangential stresses during rolling. The instrumented roll termed "SGRoll" was segmented into two parts along the axial direction in order to position the sensing elements into the roll body. An experiment was run on a laboratory rolling mill equipment using 1100 aluminum as the work material. The experimental results showed that the friction coefficient decreased gradually as the workpiece entered the roll until no slip point and then decreased rapidly as the work piece exited the roll.
\end{abstract}

Keywords: Friction, Force, Coefficient, SGRoll, Roll Angle

\section{INTRODUCTION}

The mechanics of friction are complex, and the fundamentals of the phenomenon have been the subject of considerable study. Nevertheless, very little is known that would facilitate the formulation of the exact functional relationship between the friction force and the process variables. With the progress made in the understanding of metal forming processes and with the development of mathematical analysis tools, the role of friction force becomes more obvious. While there is a reasonable under- standing of the mechanisms of friction at the roll work piece interface during rolling, the actual magnitude of the friction force is still largely a matter of conjecture. Several attempts have been made by researchers including Siebel and Lueg (1933), Rooyern and Backofen (1957), Al-Salehi et al. (1973), Truncer and Dean (1987), Jeswiet (1993), Lagergren et al. (1997), and Tieu and Liu (2004) to measure the magnitude of friction within the contact region during rolling process. Most methods are based on measuring pin or pins mounted on the sur- 


\section{Davis et al.}

face of the rolls. The normal pressure and the frictional stress are measured by pins embedded in the work roll and transducer combinations. The pins are mounted in radial and oblique directions with respect to the axis of the roll. During rolling, the pins sense and pick a pressure signal, which is relayed to the transducer. The transducer then converts the signal into a detectable output. The output is converted into force parameters. A force analysis of the signals from the pins, and the transducers yields the roll pressures and the frictional stresses. The coefficient of friction is derived from these two quantities.

From the above friction coefficient measuring techniques, it was clear that the pin measuring technique received much attention. Nevertheless, the possibility of lateral deflections of the oblique pin tend to create contact between the pin and the inside surface of the hole. Consequently, the output of the transducer may include unpredictable errors into the measurement of the frictional stress. For the frictional stress measurement, the oblique pin needed some allowance for lateral deflections. Also, the high accuracy of measurement in the placement of the oblique pin leads to sophistication of the instrumented roll and for that matter the experimental set up. Consequently, the cost of measurement, using the pin technique would be very high thereby rendering the pin technique uninteresting and unattractive. The objective of this work is to modify the pin measuring technique through design changes of the instrumented roll. The improved instrumented roll referred to as the "SGRoll" would be less expensive, would use simpler sensors, and would make provision for lateral deflection.

\section{Design Features of SGRoll}

Dimensions of the SGRoll were chosen to fit a two high roll laboratory rolling mill equipment. Details of the experimental setup are presented in section 4. As shown in Fig. 1, the SGRoll is segmented into two parts along the axial direction in order to embed sensing elements into the roll body. There were two sensing elements, namely, the radial sensing element and the tangential sensing element. The radial sensing element comprised of a conical pin, a spherical ball, and a radial rectangular bar. The tangential rectangular bar made up the tangential sensing element. Strain gauges were bonded to the radial rectangular bar and the tangential rectangular bar for the measurement of rolling forces. Two holes were drilled on either side of the SGRoll to accommodate the transmitting wires from the strain gages. The next section discusses the rolling forces measurement principles.

\section{Normal Pressure and Frictional Stress Measurement Principles}

Fig. 2 shows a schematic diagram of the radial sensing element. A conical pin was fitted radially into the SGRoll with the end flushing with the SGRoll surface, making contact with the work part during rolling operation. Consequently, the resulting normal force $\left(F_{R}\right)$ was transmitted to the radial rectangular bar through the conical pin and the spherical ball. The transmitted normal force caused a strain in the radial rectangular bar, which was measured with the strain gage. The output of the strain gage was sensed and picked up by the data acquisition system. Fig. 3 shows a schematic representation of the tangential sensing element. A tangential rectangular bar, which fitted radially into the SGRoll was located on the same axis as the conical pin, but a few inches apart. The end of the tangential rectangular bar, which flushed with the SGRoll surface made contact with the part during rolling. As the part was squeezed and pulled along the direction of the SGRoll, the frictional force $\left(\mathrm{F}_{\mathrm{T}}\right)$ caused a strain in the tangential rectangular bar and was detected with the strain gage.

The strain gage was calibrated in order to convert strain output signals to equivalent force measurements. Since the rectangular bars have uniform cross sectional areas, and also the material property was the same for all the parts of the SGRoll, the frictional stress resulted in a proportional relationship between the strain 


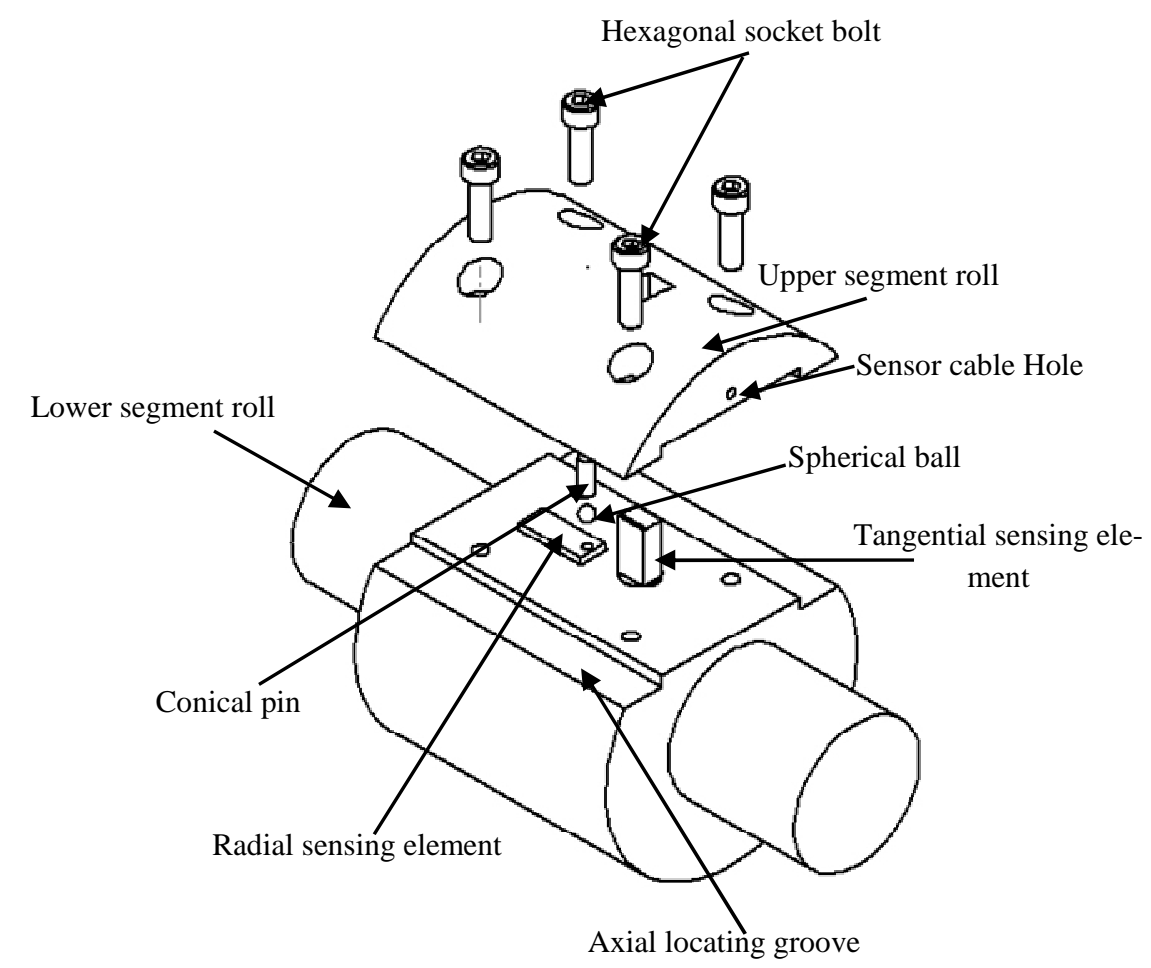

Fig. 1: Design of the SGRoll

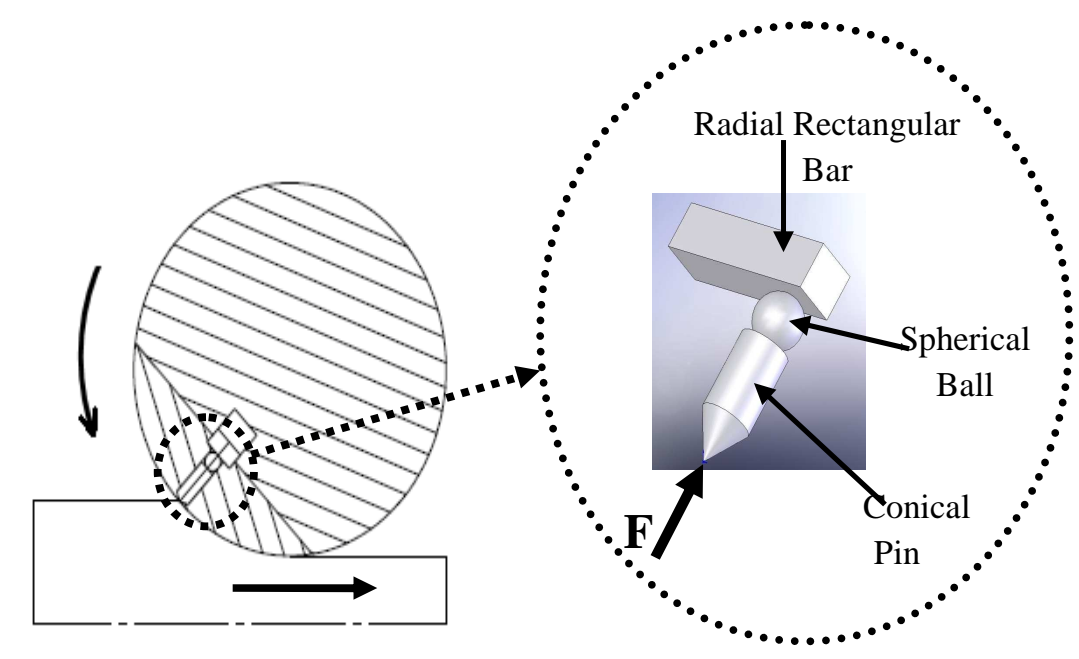

Fig. 2: Normal force measurement principle 


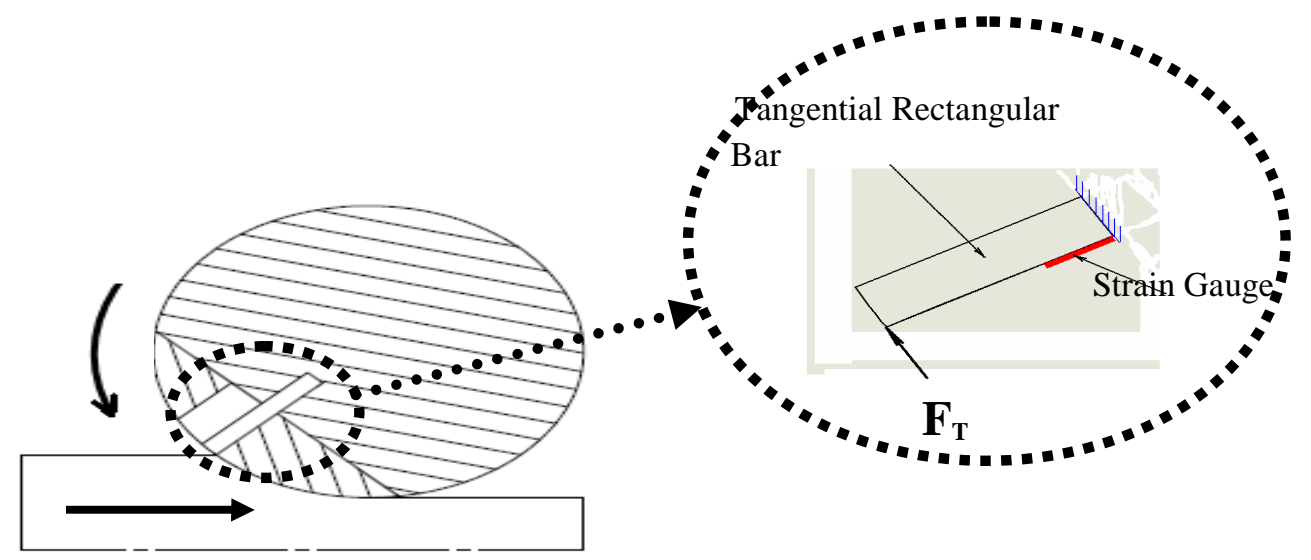

Fig. 3: Frictional force measurement principle

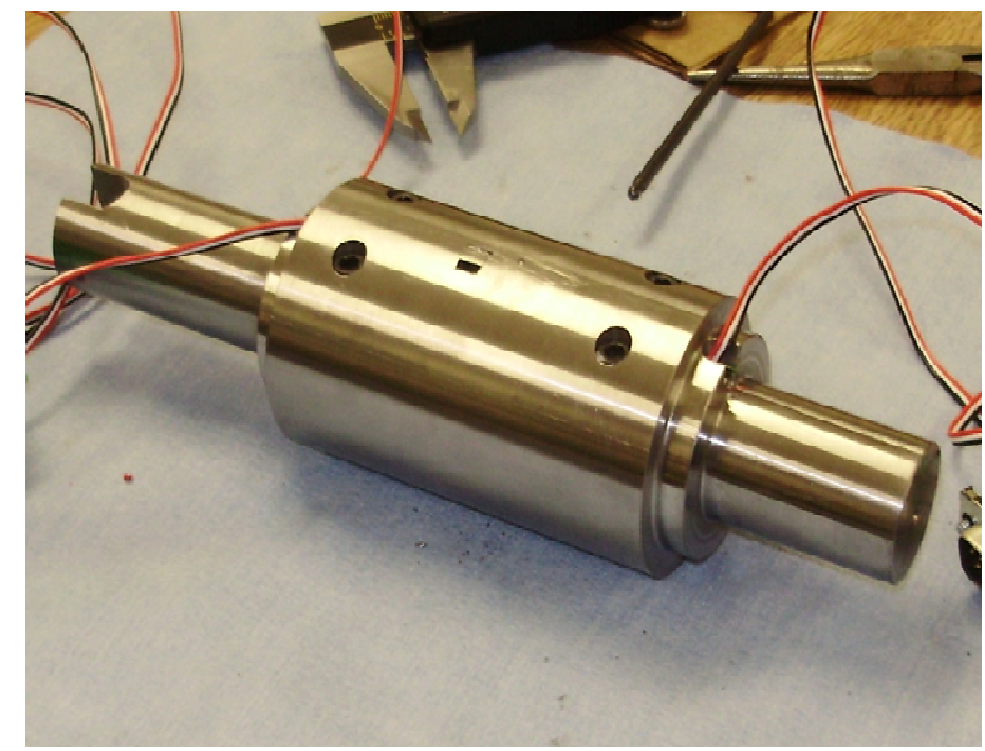

Plate 1: Photograph of the SGRoll

gage voltage output and the stresses, the constant of proportionality being the conversion factor. The material used in manufacturing the SGRoll is 4140 heat-treated steel. Plate 1 is a photograph of the manufactured SGRoll. The performance of the SGRoll compares with otherpin measuring techniques whose designs are much more sophisticated and costly. 


\section{Experimental Work}

Tests were carried out on a two-high experimental rolling mill powered by a variable speed motor of 0.5 horse power at the roll. The maximum rolling force and the rotational speed were $30,000 \mathrm{Ib}(133.5 \mathrm{KN})$ and $20 \mathrm{rpm}$, respectively. The rolls were of dimensions 2 inch $(50.8 \mathrm{~mm})$ diameter and 4 inch $(101.6 \mathrm{~mm})$ length. The roll gap, which controlled the exit thickness of the workpiece was set using an adjustable screw system. The maximum available gap was 0.5 inches $(12.7 \mathrm{~mm})$. The data acquisition system included System 5000 Scanner and Strain Smart Software. The System 5000 Scanner picked up signals through the transmitting wires while the Smart Strain Software processed the data. The sampling rate was $100 \mathrm{~Hz}$. The strain gage transmitting wires from the SGRoll were connected to the input of the System 5000 Scanner. Plate 2 is a photograph of the experimental set up. During rolling, the SGRoll and the lower roll progressively squeezed the workpiece as it entered and exit the rolls. As a result, the conical pin caused a strain in the radial rectangular bar. Simultaneously, the tangential rectangular bar caused a strain in the tangential rectangular bar. The strain in the respective strain gages changed the output of the wheatstone bridge set up enclosed in the System 5000 Scanner.

\section{Experimental Procedure}

Aluminum alloy 1100 with dimensions of 0.375-inch $(9.525 \mathrm{~mm})$ thick and 1-inch $(25.4$ $\mathrm{mm}$ ) wide was used in the experiments. Design calculations coupled with experimental trials revealed that

(i) $20 \%$ reductions was not feasible with the

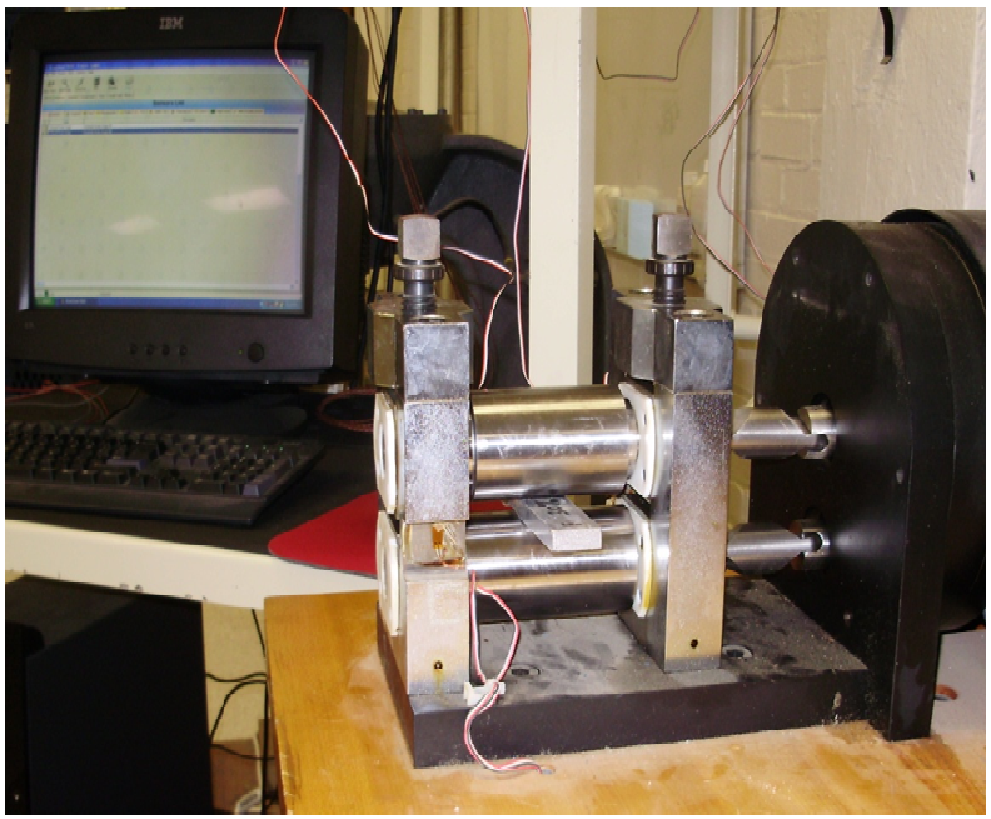

Plate 2: Photograph of experimental set up 


\section{Davis et al.}

data acquisition system for the maximum sampling rate of $100 \mathrm{~Hz}$;

(ii) $30 \%$ reductions was possible with the data acquisition system for the maximum sampling rate of $100 \mathrm{~Hz}$;

(iii) $40 \%$ reduction was not successful in one pass rolling due to the roller speed. Consequently, the reduction was fixed at $30 \%$.

The experimental steps are as follows:

1) Cut the specimen to size and chamfer one end of specimen to facilitate entry into the rolls.

2) Set the roll gap to the exit thickness.

3) Turn on the main power to the control panel. Start hydraulic power unit and set speed to conform to the selected speed. In this experiment, the selected speed was $5 \mathrm{rpm}$.

4) Select FWD knob of the roll mill. Feed the specimen through the roll gap and select STOP knob of the roll mill. At this point ensure that the axis of the conical pin and tangential rectangular bar are well marked or located.

5) Check the SGRoll transmitting wire readings to ensure that there is no strain on the strain gage. Connect the transmitting wires to the input socket of the System 5000 Scanner. Once again check strain gage readings at the input socket. Turn ON System 5000 Scanner.

6) Initiate Smart Strain software. Set the sampling rate. In this experiment, the sampling rate was set at $100 \mathrm{~Hz}$. Match System 5000 Scanner input channel to Smart Strain software channel. In this experiment, channel 1 is for the radial rectangular bar and channel 2 is for the tangential rectangular bar. Perform net zero strain and shunt calibration. At this point the data is ready to be col- lected.

7) Hit START on the recording pop up menu. Then select FWD knob of the roll mill to start the rolling. As soon as the axis of the conical pin exits the contact region, hit STOP on the recording pop up menu, and then select STOP on the roll mill. Smart Strain software prepares the output file and presents it in Excel format.

The measured data from the experiment is presented in Fig. 4. From Fig. 4, friction and slipping between the work and roll interface caused the radial strain to increase continuously as work entered the roll until maximum radial strain was attained at a time of about $0.45 \mathrm{sec}$ onds. Thereafter, the friction and slipping between the work and roll interface caused the radial strain to decrease rapidly until the work exited the roll. Also, the tangential strain decreased continuously as the work entered the roll until about half way through the contact region. The tangential strain remained stationary for a few seconds and thereafter decreased rapidly as the work part exited the roll. The next sections discuss the extraction of the dynamic friction coefficient from the measured data.

\section{ANALYSIS AND DISCUSSION OF RESULTS}

Table 1 shows the results obtained from the experiment. The normal force and frictional force was evaluated using the strain - force calibration charts. Subsequently, the friction coefficient was calculated as the ratio of frictional force to the normal force. The friction coefficient derived from the experiment is plotted in Fig. 5. The plot indicates that as the workpiece made contact with the roll, the friction coefficient decreased slowly from a maximum value of about 0.11 until the point of noslip. At this point, the friction coefficient decreased rapidly as the workpiece exited the roll. This occurrence is consistent with the principle of physics in that friction force between surfaces in contact decreased continuously as the 


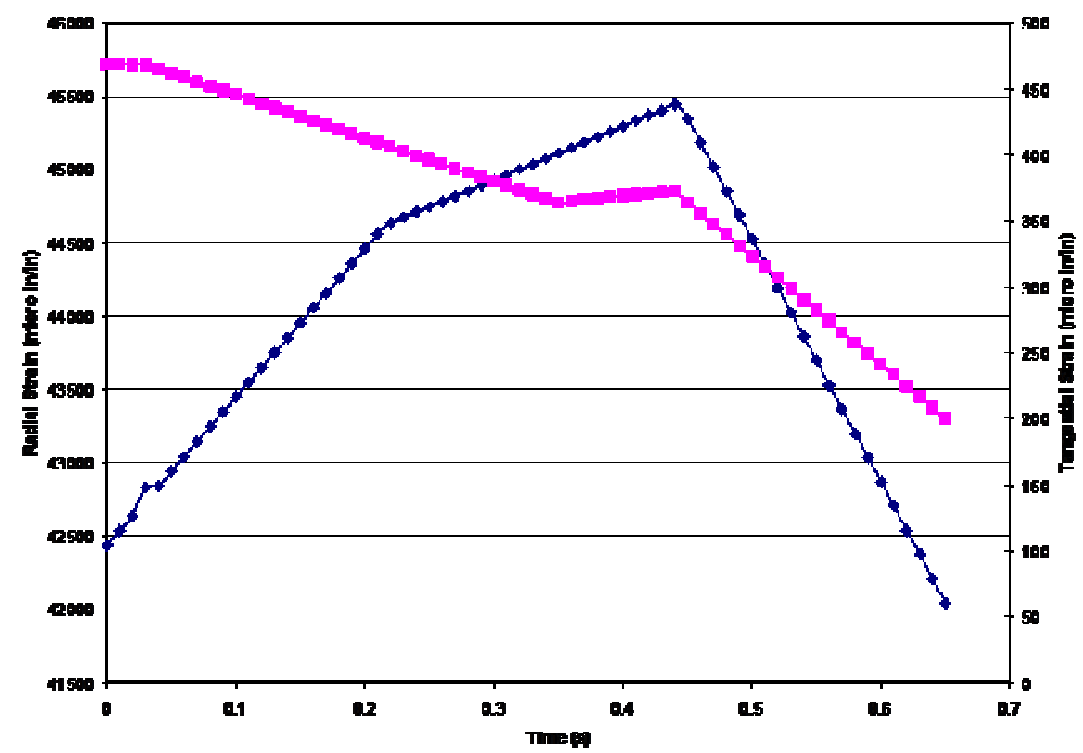

Fig. 4: Plot of measured data

Table 1: Extraction of measured friction coefficient

\begin{tabular}{|c|c|c|c|c|}
\hline \multirow[b]{2}{*}{ Time (s) } & \multicolumn{3}{|c|}{ Strain - force calibration } & \multirow[b]{2}{*}{$\boldsymbol{\mu}$} \\
\hline & $\theta(\mathbf{r a d})$ & Normal force (lbf) & Friction force (lbf) & \\
\hline 0.00 & 0.337 & 462 & 49 & 0.1062 \\
\hline 0.05 & 0.311 & 468 & 48 & 0.1034 \\
\hline 0.10 & 0.285 & 473 & 47 & 0.0986 \\
\hline 0.15 & 0.259 & 479 & 45 & 0.0939 \\
\hline 0.20 & 0.233 & 484 & 43 & 0.0893 \\
\hline 0.25 & 0.207 & 487 & 42 & 0.0853 \\
\hline 0.30 & 0.181 & 489 & 40 & 0.0814 \\
\hline 0.35 & 0.155 & 491 & 38 & 0.0777 \\
\hline
\end{tabular}

part transitional from quasi static to dynamic mode. Besides, the experimental result matches with Tieu and Liu's experimental results (Tieu, 2004). Meanwhile, Lenard (2002) has described Tieu and Liu's experiment as elaborate and costly.

\section{CONCLUSION}

A simple friction coefficient measurement technique was developed and tested. This measurement technique could complement the efforts of the industry in predicting the power and energy requirements for the rolling process. The fol- 


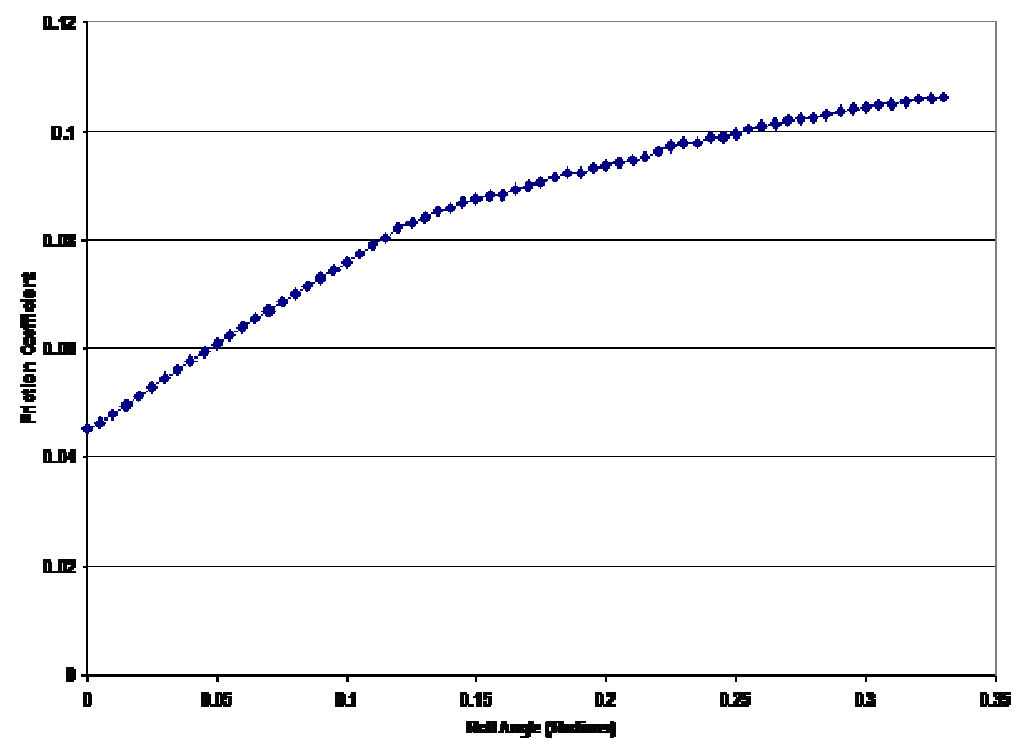

Fig. 5: Measured friction coefficient

lowing conclusion and suggestions can be made based on the results obtained:

1. The friction coefficient decreased slowly from the entry point until the point of no slip, and then decreased rapidly to the exit point. This happening is consistent with the physics in the contact region.

2. The experimental setup may be improved by extending the angles of rotation of the roll. The transmitting wires used in the experiment had the tendency of limiting the number of angular rotations. Further design work needs to be done to extend the range of the data collected. This may include the use of a wireless SGRoll.

\section{REFERENCES}

Al-Salehi, F. A. R., Firbank, T. C. and Lancaster, P. R. (1973). "An experimental determination of the roll pressure distribution in cold rolling, International Journal of Mechanical Science, 15: 693-710.

Avitzur, B. (1968). Metal Forming, Process and Analysis, McGraw-Hill Book Company, New York, pp 475-476.

Davis, F. (2010). "Effects of variable friction coefficient on the pressure distribution between a rolling cylindrical element and a deformable flat surface", $\mathrm{PhD}$ Dissertation, North Carolina A \& T State University, Greensboro.

Dieter G. E.(1976), Mechanical Metallurgy, Materials Science and Engineering Series, McGraw-Hill Book Company, New York, Second Edition, pp. 613-614.

Jeswiet, J. and Nyahumwa, C. (1993), A sensor for measuring metal deformation interface forces, Journal of Materials Processing 
Technology, 39: 251-268.

Keife, H. and Sjogren, C. (1994). "A friction model applied in the cold rolling of aluminum strips, Wear, 60: 137-142.

Lagergren, J. (1997). "Friction evaluation in hot strip rolling by direct measurement in the roll gap of a model duo Mill, Journal of $\mathrm{Ma}$ terial Processing and Technology, 70: 207214.

Lenard, J. G. (2002). Material Forming Science and Practice, Elsevier Science Ltd., New York, pp. 90-91.

Lenard, J. G. and Malinowski, Z. (1993). "Measurement of friction during warm rolling of aluminium," Journal of Materials Processing Technology, 39: 357-371.

Liu, Y. J., Tieu, A. K., Wang, D. D. and Yuen,
W.Y.D. (2001). "Friction measurement in cold rolling," Journal of Materials Processing Technology, 111: 142-145.

Roberts, C. D. (1997). "Mechanical principles of rolling," Journal of Iron and Steel, 24: 113 -114 .

Rooyen, G. T. V. and Bachofen, W. A. (1957). "Friction in Cold Rolling," Journal of the Iron and Steel Institute, 6: 235-244.

Tieu, A. K. and Liu, Y. J. (2004). "Friction variation in the cold rolling process," Tribology International, 37: 177-183.

Truncer, C. and Dean, T. A. (1987). "A new pin design for pressure measurements in metal forming processes," International Journal of Machine Tools Manufacturing, 27(3): 325 331 . 